\title{
Evolution de notre compréhension du système de mousson ouest-africain
}

\section{Résumé}

Depuis l'expérience internationale pionnière GATE en 1974, notre documentation et notre compréhension du système de mousson africaine avaient évolué relativement lentement. Comment notre vision de cette mousson ressort-elle après les $\mathbf{1 0}$ ans du projet AMMA ? Si les principaux acteurs de ce système couplé atmosphère-océancontinent étaient déjà bien connus, AMMA a permis d'approfondir le détail des processus en jeu et de leur couplage. D'une vision statique de ce système émerge petit à petit une vision plus dynamique où intervient désormais l'ensemble du globe : l'Afrique en entier, les latitudes tempérées, la mousson indienne et même toute la ceinture tropicale. Une voie est ainsi ouverte pour comprendre sa variabilité intrasaisonnière.

\section{Abstract}

\section{Progress on understanding of the} West African Monsoon system

Since the pioneering GATE international field experiment in 1974, little progress had been made on the documentation and understanding of the West African Monsoon system. Ten years after the AMMA project was initiated, how has our knowledge of this monsoon evolved? Although the main actors of this atmosphereocean-continent coupled system were already known, AMMA has allowed us to study thoroughly the details of the processes at play and their coupling. Starting from a static view of the system, a more dynamical view is now emerging, involving the whole Earth system: the whole African continent, mid-latitudes, the Indian monsoon and the entire tropical belt. This has opened new avenues of investigation into the intraseasonal variability of the West African monsoon.

\author{
Jean-Philippe Lafore(1), Nicole Asencio(1), Dominique Bouniol(1), \\ Fleur Couvreux (1), Cyrille Flamant(2), Françoise Guichard(1), \\ Nicholas Hall(3), Serge Janicot(4), Cécile Kocha(1), \\ Christophe Lavaysse(2-5), Stéphanie Leroux(6), Emmanuel Poan(1), \\ Philippe Peyrillé(1), Rémy Roca(7), Romain Roehrig(1), Frank Roux(8) \\ et Frédérique Saïd(8) \\ (1) CNRM-GAME, CNRS et Météo-France, \\ 42 avenue Coriolis - 31057 Toulouse \\ (2) LATMOS, CNRS et Université Pierre et Marie Curie, Paris, France \\ (3) LEGOS, CNRS, CNES, IRD et Université de Toulouse, Toulouse, France \\ (4) LOCEAN/IPSL IRD, Paris, France \\ (5) McGill University, Montréal, QC, Canada \\ (6) Department of Atmospheric and Environmental Sciences, \\ SUNY Albany, NY, États-Unis \\ (7) LMD, CNRS, École normale supérieure, École polytechnique \\ et Université Pierre-et-Marie-Curie, Paris, France \\ (8) LA, Université de Toulouse et CNRS, Toulouse, France
}

\section{À} la fin $\mathrm{du} \mathrm{XX}^{\mathrm{e}}$ siècle, la mousson d'Afrique de l'Ouest (MAO) restait l'un des systèmes de mousson les moins bien documentés. L'essentiel de nos connaissances reposait sur l'expérience internationale GATE (GARP(1) Atlantic Tropical Experiment) de 1974, complétée par quelques campagnes spécifiques comme COPT81 ${ }^{(2)}$ (Sommeria et Testud, 1984) ou HAPEX-Sahel ${ }^{(3)}$ (Goutorbe et al., 1994) et par l'expérience des prévisionnistes. La première phase du projet international AMMA (2002-2010) a apporté un souffle nouveau à l'étude de la MAO. Partant du schéma conceptuel que nous avions au début des années 2000, cet article présente celui qui émerge aujourd'hui grâce aux travaux réalisés dans le cadre d'AMMA. Pour compléter cet article, par essence didactique et non exhaustif, nous conseillons au lecteur intéressé la lecture de la synthèse de Lafore et al. (2011).

(1) GARP : Global Atmospheric Research Program. (2) COPT81: Convection profonde tropicale. (3) HAPEX-Sahel : Hydrological and Atmospherical Pilot Experiment for the Sahel.

\section{Schéma conceptuel à la fin du $X^{\mathrm{e}}$ siècle}

Une mousson est un système couplé atmosphère-océan-continent. Un schéma synthétisant les composantes essentielles de la MAO est proposé sur la figure 1. Plusieurs spécificités géographiques en font un système unique, avec comme seul éventuel analogue la mousson australienne, qui concerne cependant un continent moins étendu. L'Afrique de l'Ouest est située à proximité de l'équateur, ses reliefs sont peu élevés, elle est entourée d'eau sur trois de ses façades (Atlantique au sud et à l'ouest, Méditerranée au nord) et englobe, au nord, le plus grand désert du monde, le Sahara. Sa configuration quasi zonale en fait, au premier ordre, un système bidimensionnel.

$\mathrm{Au}$ printemps, le continent se réchauffe alors que les eaux du golfe de Guinée se refroidissent, formant une langue d'eau froide étudiée par Caniaux et al. dans ce numéro, p. $17-$ 24. Sous l'effet de ce contraste, les alizés de sud-est traversent l'équateur, 


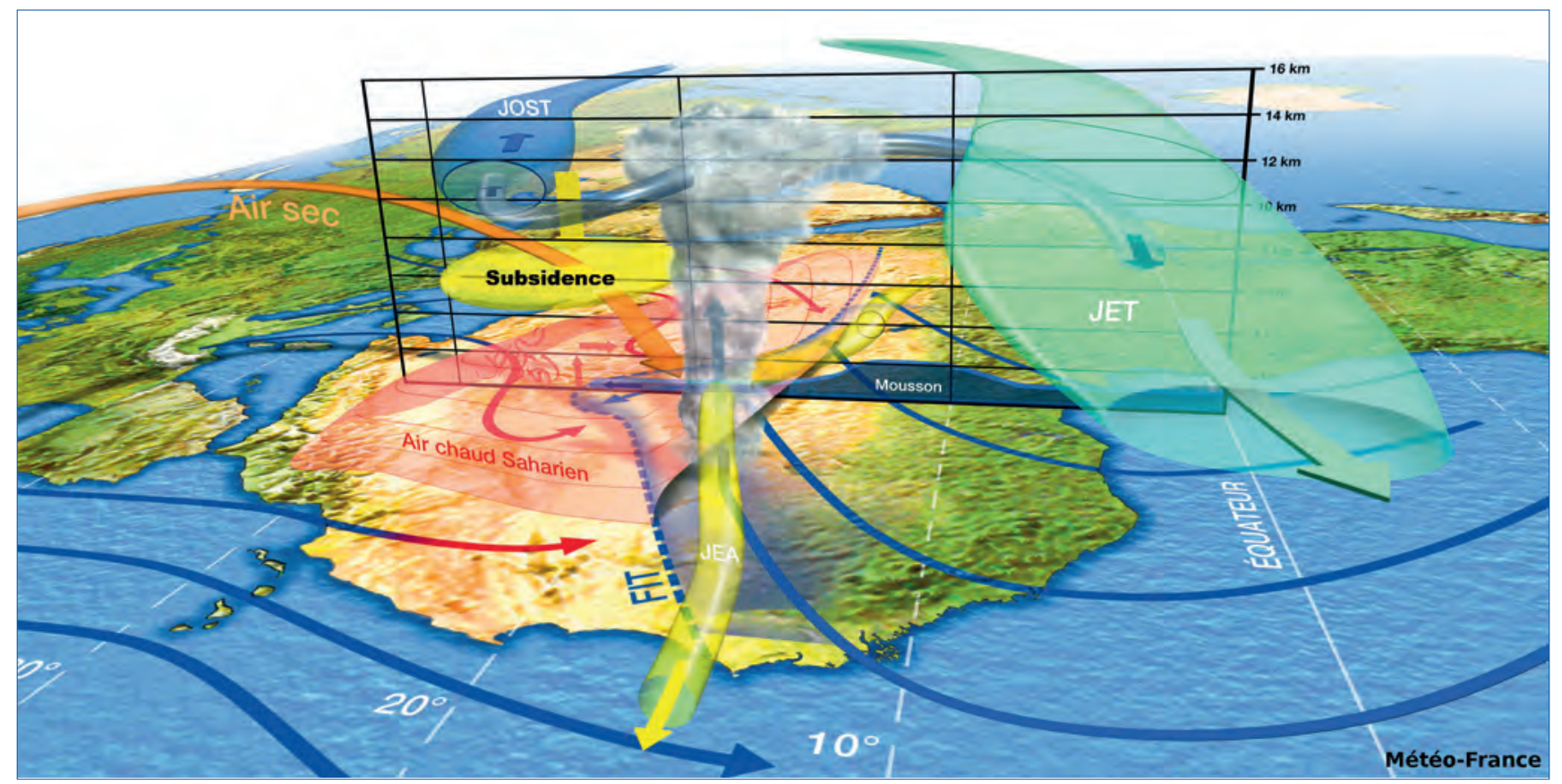

Figure 1 - Schéma synthétisant les composantes essentielles de la mousson de l'Afrique de l'Ouest.

pivotent en un vent de sud-ouest et pénètrent sur le continent où ils forment le flux de mousson dans les basses couches de l'atmosphère (flèches bleues). Le réchauffement continental est maximal sur le Sahara où s'installe un immense dôme d'air chaud, pouvant atteindre une épaisseur de 4-5 km, et appelé dépression thermique saharienne (DTS). Cette dépression de surface permet la convergence, au niveau du front intertropical (FIT, ligne pointillée bleue, vers $20^{\circ} \mathrm{N}$ ), entre le flux de mousson frais et humide et les alizés de nord, chauds et secs (harmat$\tan$ ), en provenance de la Méditerranée et de l'Atlantique nord.

Le contraste entre ces deux masses d'air induit dans la bande sahélienne un fort gradient méridien de température, responsable de la formation du jet d'est africain (JEA, tube jaune). L'existence du JEA en moyenne altitude $(600-700 \mathrm{hPa})$ favorise le développement des ondes d'est, documentées notamment pendant l'expérience GATE de 1974. La convection est favorisée autour du thalweg des ondes d'est. Globalement, elle s'organise au sud du FIT et du JEA, là où l'instabilité convective est maximale, formant la zone de convergence intertropicale (ZCIT), située vers $10-15^{\circ} \mathrm{N}$ en juillet et août. La combinaison du fort cisaillement de vent dans la basse atmosphère et de l'air sec surplombant le flux de mousson favorise la formation de lignes de grains se propageant rapidement vers l'ouest grâce à de puissants courants de densité (schéma conceptuel de Lafore et Moncrieff, 1989). Dans la haute troposphère, la ZCIT induit un écoulement divergent anticyclonique qui renforce au sud le jet d'est tropical (JET, tube vert) et au nord le jet d'ouest subtropical (JOST, tube bleu). Le JET correspond à la branche d'altitude de la cellule de Walker, initiée par la mousson indienne et s'atténuant sur l'Afrique. Enfin, la DTS est surmontée au-dessus de $500 \mathrm{hPa}$ par une forte subsidence (zone jaune) correspondant à la circulation indirecte de la cellule de Hadley locale, empêchant le développement de la convection profonde sur le Sahara.

\section{Contrôle \\ de la mousson par la surface via la couche limite}

Le schéma précédent peut donner l'impression fausse que l'essentiel de l'eau indispensable aux pluies de mousson est importée de l'océan, par le flux de mousson. En fait une grande partie provient du recyclage de l'eau des précipitations sur le continent, c'est-à-dire de l'évaporation au-dessus des sols précédemment arrosés par les pluies. Ce processus est particulièrement fort sur l'Afrique et son étude était l'un des objectifs du projet AMMA. Son étude, résumée par Peugeot et al. (dans ce numéro, p. 55-63) a permis de quantifier sa contribution au cumul saisonnier des précipitations.

Une question clé est de connaître les facteurs qui conditionnent la pénétration des pluies de mousson sur le continent et donc la "qualité » de la mousson. Les études précédentes ont accordé un rôle majeur à la température dans le golfe de Guinée dont le refroidissement au printemps favorise cette pénétration. En complément l'attention s'est portée sur la Méditerranée. Comme montré par les travaux de modélisation idéalisée de Peyrillé et al. (2007), le réchauffement estival de la Méditerranée réduit le contraste thermique avec l'Afrique du Nord et donc l'advection d'air frais et humide (ventilation) au nord de la MAO. La DTS s'en trouve renforcée d'où une pénétration plus forte de la mousson.

Outre les températures des océans entourant l'Afrique, la forte variation méridienne des conditions de surface sur l'Afrique de l'Ouest est également un élément majeur pour expliquer la circulation observée de la MAO (figure 1). Du sud au nord, la côte atlantique $\left(\sim 5^{\circ} \mathrm{N}\right)$ avec un climat humide soudanien laisse progressivement la place au Sahara, l'un des déserts les plus arides, avec une zone de transition semidésertique, le Sahel $\left(\sim 10-20^{\circ} \mathrm{N}\right)$. Les sols sont donc de plus en plus chauds et secs, permettant aux flux de chaleur sensible de prendre le pas sur les flux de chaleur latente. Il en résulte des couches limites très épaisses, moins nuageuses 


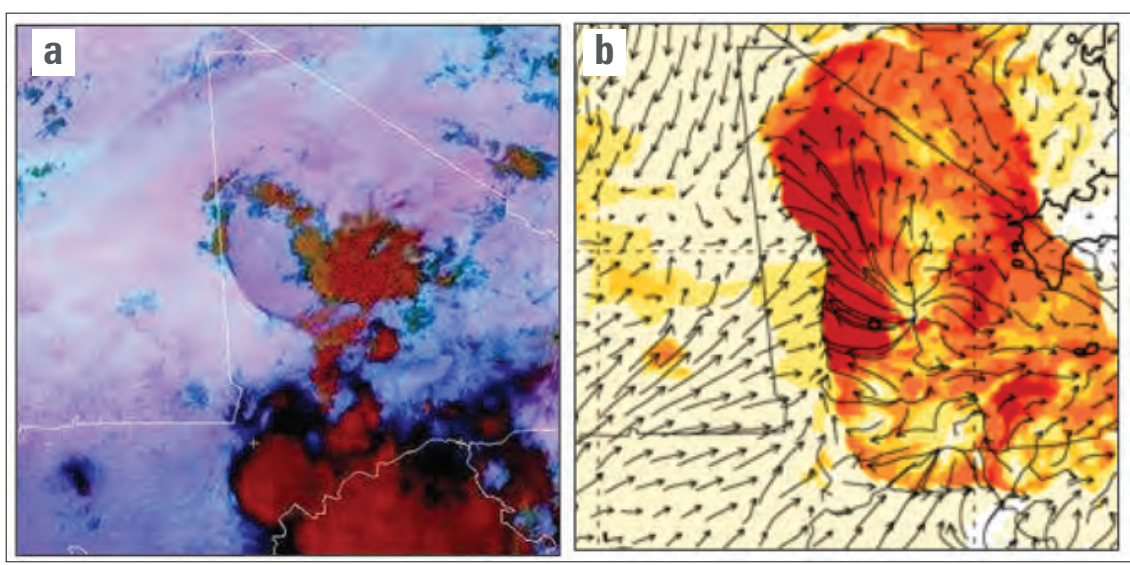

Figure 2 - (a) Observation par MSG(1) d'un soulèvement de poussières (zone colorée en magenta) par le courant de densité associé à un système convectif (zones rouges sombre) sur le nord du Mali, le 23 juin 2011 à 00 h UTC durant la campagne FENNEC.

(b) Prévision par AROME à $5 \mathrm{~km}$ de résolution (vent et extinction par les poussières au premier niveau) à 30 heures d'échéance. Le domaine couvert est de $12^{\circ}$ carré.

et avec des bases de nuages élevées dans la DTS. La couche limite joue alors le rôle de courroie de transmission entre la surface et l'atmosphère, permettant l'établissement d'une zone de fort gradient méridien de température (baroclinie) dans la basse atmosphère, maximale sur le Sahel, et couplée à un gradient méridien inverse d'humidité. AMMA a permis de documenter le fonctionnement de ces couches limites contrastées (Gounou et al., 2012), dont le développement vertical est favorisé par le cisaillement entre le flux d'harmattan et le flux de mousson (Canut et al., 2011). Le fort cycle diurne de ces couches limites, couplé à une forte oscillation de la position du FIT, a été étudié. Il est associé à l'occurrence d'un maximum du jet de basse couche au matin favorisant la progression de la mousson vers le nord (Lothon et al., 2008). La dynamique de la couche limite joue un rôle important dans la phase d'installation de la mousson, en mélangeant le flux de mousson avec l'air sec qui le surplombe. Ce processus de dilution du flux de mousson retarde sa pénétration sur le continent.

\section{Convection et nuages}

Le dispositif expérimental déployé durant la campagne de terrain d'AMMA (Lebel et al., 2010) a permis la documentation de nombreux systèmes convectifs qui a confirmé le schéma conceptuel des systèmes convectifs propagatifs, de type ligne de grains, tel qu'issu de la campagne COPT81. L'ensemble de ces études fait ressortir l'importance de l'air sec sur l'Afrique de l'Ouest, et notamment l'existence d'intrusions d'air sec extratropical dans le JEA (Roca et al., 2005). Cet air sec est responsable de la formation d'intenses courants de densité qui contribuent à organiser la convection. Cependant, à plus grande échelle, AMMA a révélé que ces zones sèches ont tendance à inhiber l'activité convective sur l'ensemble de la bande sahélienne (Roca et al., 2012). Au-delà de ces cas d'étude, des climatologies des différents types de systèmes convectifs (Fiolleau et al., 2009) ont été établies à l'aide de Météosat. Elles ont permis de connaître la représentativité de chaque cas d'étude en fonction de la région et de l'environnement. Ainsi les lignes de grains ne sont pas le seul type d'organisation de la convection, et notre attention s'est également portée sur l'étude de systèmes moins organisés et de durée de vie plus courte qui jouent également un rôle important dans la MAO.

L'étude des interactions de la convection avec les échelles supérieures et de son couplage avec d'autres processus (surface, poussières) a été une évolution marquante. Ainsi, les systèmes convectifs sont souvent couplés à la grande échelle, comme les ondes d'est ou les pulsations de mousson (Barthe et al., 2010 ; Cuesta et al., 2010), sur laquelle ils rétroagissent. Par exemple, le transport vertical de vent méridien par la convection renforce le thalweg des ondes d'est. À méso-échelle, les hétérogénéités d'humidité du sol sont apparues importantes pour le déclenchement de la convection au Sahel (Guichard et al., ce numéro p. 25-32). Le couplage de modèles à haute résolution, tel qu'AROME ${ }^{(2)}$, avec un module de poussières désertiques, a permis de simuler la génération de panaches de poussières par les courants de densité convectifs (figure 2). De tels événements peu ou pas précipitants sur le Sahel sont fréquents avant le démarrage de la mousson et représentent une source importante de poussières expliquant leur maximum d'occurrence observé en juin.

La campagne AMMA et sa composante américaine NAMMA $^{(3)}$ de la NASA (National Aeronautics and Space Administration) ont également abordé l'étude de la cyclogenèse tropicale dans la zone du Cap-Vert, en aval de la MAO. Celle-ci résulte d'interactions complexes entre la convection, une onde d'est et l'écoulement synoptique au-dessus de l'Atlantique tropical est (Arnault et Roux, 2009, 2010 ; Schwendike et Jones, 2010). Ces travaux soulignent le rôle joué par les circulations synoptiques issues de la dépression thermique saharienne : la circulation anticyclonique dans la moyenne troposphère issue de la DTS peut inhiber la cyclogenèse alors que la circulation cyclonique en basse couche issue de la DTS lui est favorable.

La convection est la source principale de nuages à longue durée de vie sous les tropiques. Leur contribution au bilan énergétique via leur impact radiatif est importante mais mal connue. Les mesures radar, lidar et in situ d'AMMA ont permis de caractériser les propriétés macroscopiques et microphysiques des enclumes engendrées par la convection (Bouniol et al., 2010) sur l'Afrique de l'Ouest. Par ailleurs, les distributions verticale et méridienne de l'occurrence des nuages et leur évolution saisonnière ont pu, pour la première fois, être documentées, notamment grâce aux capteurs actifs des satellites de l'A-Train. La figure 3 représente l'occurrence des nuages observés et associés à la migration de la ZCIT (zone nuageuse la plus profonde) du golfe de Guinée $\left(0-5^{\circ} \mathrm{N}\right)$ en mai, jusqu'au Sahel $\left(10-15^{\circ} \mathrm{N}\right)$ en août. Un élément remarquable est la présence récurrente de nuages de miniveau $(5-7 \mathrm{~km})$ sur tout le transect méridien et en particulier au-dessus du désert du Sahara $\left(20-30^{\circ} \mathrm{N}\right)$. À l'aide d'observations de surface, Bouniol et al. (2012) ont montré le fort impact radiatif de ces nuages dont la

(1) MSG : Meteosat Second Generation.

(2) AROME : Application de la recherche à l'opérationnel à méso-échelle.

(3) NAMMA : NASA African Monsoon Multidisciplinary Analyses. 


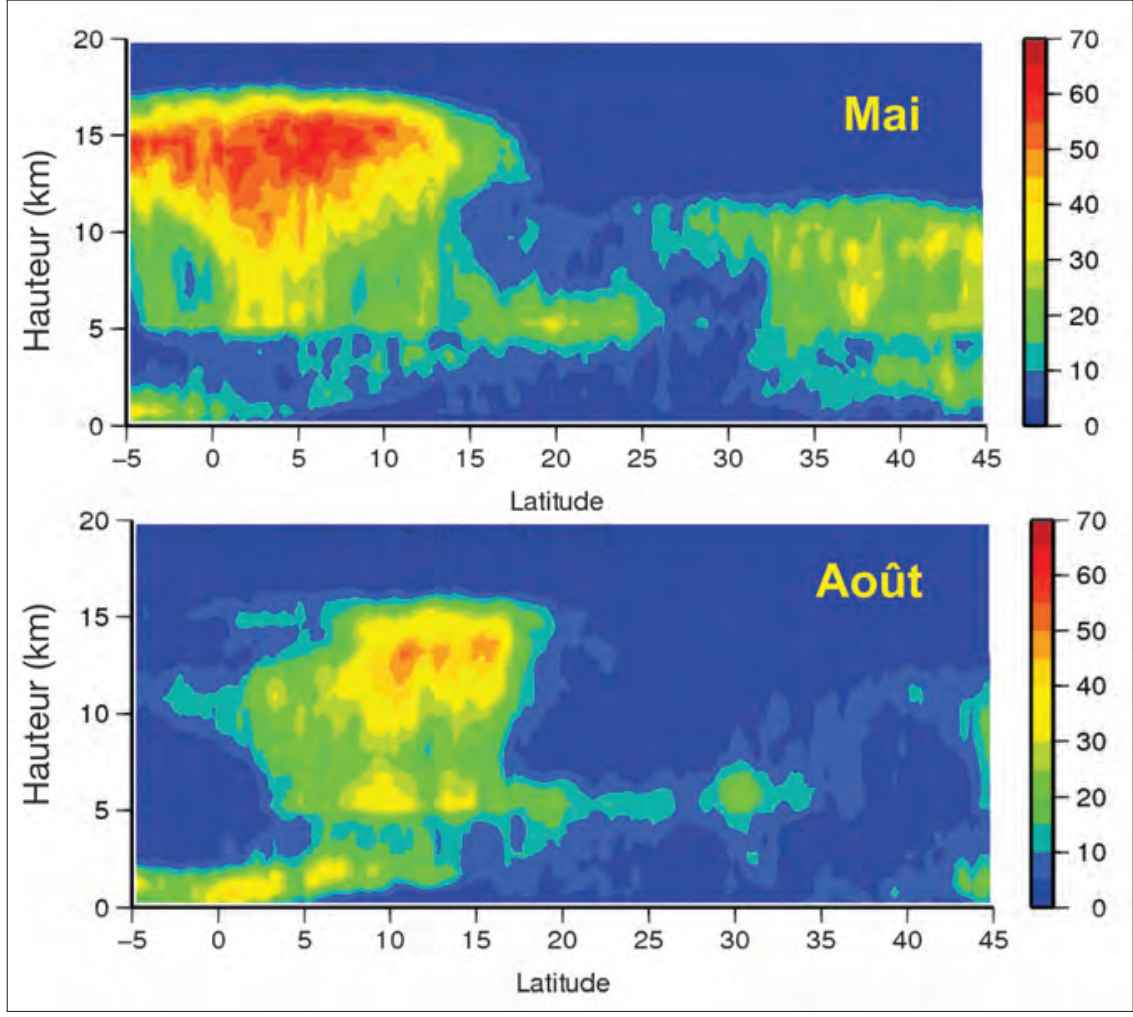

Figure 3 -

Coupe verticale méridienne de la fréquence (\%) d’occurrence de nébulosité pour les mois de mai et août 2008.

représentation reste un défi majeur des paramétrisations physiques utilisées dans les modèles de prévision ou de climat.

\section{L'aspirateur Dépression thermique saharienne}

AMMA a placé la DTS au centre de l'étude de la MAO. Ce phénomène complexe met en jeu une multiplicité d'échelles (de locale à régionale) et de processus (surface, turbulence, rayonnement, nuages, aérosols, ventilation, convection...) qui, dans leur ensemble, déterminent son intensité, sa position, son extension, son épaisseur... Différents diagnostics de la DTS ont été développés, ce qui a permis de décrire sa climatologie et sa variabilité ainsi que d'évaluer la capacité des modèles de prévision du temps et du climat à la représenter. Lavaysse et al. (2009) ont ainsi documenté son cycle saisonnier et montré qu'elle se bloque entre les massifs de l'Atlas et du Hoggar lorsque la mousson s'installe. Chauvin et al. (2010) ont mis en avant un rôle majeur des latitudes moyennes dans sa variabilité intrasaisonnière, via une modulation des advections d'air plus frais et humide (ou ventilation) par les alizés de nord de l'Atlantique et de la Méditerranée, qui façonnent sa forme, sa position et son intensité. À plus fine échelle, les études des processus dans cette couche limite épaisse ont permis de mieux comprendre sa structure multicouche, son cycle diurne et les transports verticaux qu'elle opère et qui sont mal décrits dans les modèles par les paramétrisations actuelles.

AMMA a permis d'étudier le couplage avec les poussières dans cette région, considérée comme leur première source au monde (par exemple, Lavaysse et al., 2011). Les mécanismes de soulèvement et transport des poussières par les différents acteurs de la MAO (FIT, convection, harmattan, flux orographiques...) ont été étudiés et quantifiés. L'effet radiatif des poussières correspond à un refroidissement sous le panache et un réchauffement par absorption dans sa partie supérieure, ce qui réduit l'intensité de la DTS en juin et pourrait retarder le déclenchement de la mousson (Kocha, 2011). L'intérêt croissant pour la DTS a motivé le programme international FENNEC d'étude du climat saharien. La campagne associée de juin 2011 a montré l'importance des forts vents de NE ou de NO associés aux ventilations pour le soulèvement de poussières et leur impact sur le bilan radiatif de la DTS. FENNEC a également confirmé la capacité des courants de densité convectifs à soulever les poussières, comme illustré par la figure 2 .

\section{Ondes d'est, enfin du nouveau !}

Depuis la campagne GATE, l'Afrique de l'Ouest est connue pour ses ondes d'est qui sont à l'origine d'une grande partie des dépressions et cyclones tropicaux de l'Atlantique. Elles étaient alors considérées comme un mode d'instabilité du JEA. Cette idée a été bousculée récemment par les travaux de Hall et al. (2006) qui montrent que l'instabilité est en fait trop faible et que le JEA n'a pas une extension zonale suffisante pour expliquer l'amplitude atteinte par ces ondes. Elles seraient plutôt une réponse transitoire du JEA à une excitation d'origine externe. En particulier, la convection à l'entrée est du JEA dans la région du Darfour peut provoquer une réponse ondulatoire forte lorsque le JEA y est intense et étendu en latitude (Leroux et Hall, 2009). À l'aide de simulations idéalisées, Leroux et al. (2011) suggèrent que cette excitation des ondes d'est pourrait aussi provenir des perturbations synoptiques aux latitudes moyennes.

Parallèlement, l'utilisation de la variable eau précipitable (cumul de vapeur d'eau contenue à la verticale d'un point) a permis de mettre en évidence des pulsations de mousson sur le Sahel, avec des périodes de l'ordre de 4 jours en juin, avant l'installation de la mousson. Couvreux et al. (2010) montrent qu'elles sont précédées par un gonflement de la DTS permettant une intensification et un épaississement du flux de mousson, et donc une augmentation du cumul. De telles pulsations sont en fait observées pendant toute la mousson comme l'illustre la figure 4 pour la saison 2006. Les anomalies traversent toute l'Afrique d'est en ouest $\left(\sim 9 \mathrm{~m} \mathrm{~s}^{-1}\right)$, avec une période de 5 jours, et présentent beaucoup de similarités avec les ondes d'est détectées à partir de la dynamique au niveau du JEA (700-600 hPa). Létude statistique de Poan et al. (2012) souligne que les événements humides et secs associés sont fréquents ( 3 de chaque signe par mois) et modulent fortement les précipitations. Les processus diabatiques, en particulier la forte évaporation des pluies dans l'air sec sous-nuagueux avant qu'elles n'atteignent le sol, est un élément important dans la croissance des anomalies. Cette variable apporte une vision complémentaire de la variabilité 
synoptique sur l'Afrique de l'Ouest, avec l'avantage d'avoir des signaux précurseurs forts sur l'est du Sahel, avec parfois des échelles plus lentes ( 15 jours, figure 4).

Dans cette gamme d'échelles temporelles, Mounier et al. (2007) et Mekkonen et al. (2008) mettent en évidence en été le passage d'ondes de Kelvin d'une

Figure 4 - Diagramme spatio-temporel durant la mousson 2006 (juin à septembre) de l'évolution de la distribution zonale de l'anomalie intrasaisonnière du cumul de vapeur d'eau (en $\mathrm{mm}$ ) dans la bande sahélienne $\left(12-20^{\circ} \mathrm{N}\right)$. Les périodes les plus humides (sèches) sont indiquées en bleu (rouge). Le trait oblique correspond à une vitesse de propagation vers d'ouest de $9 \mathrm{~m} \mathrm{~s}^{-1}$.

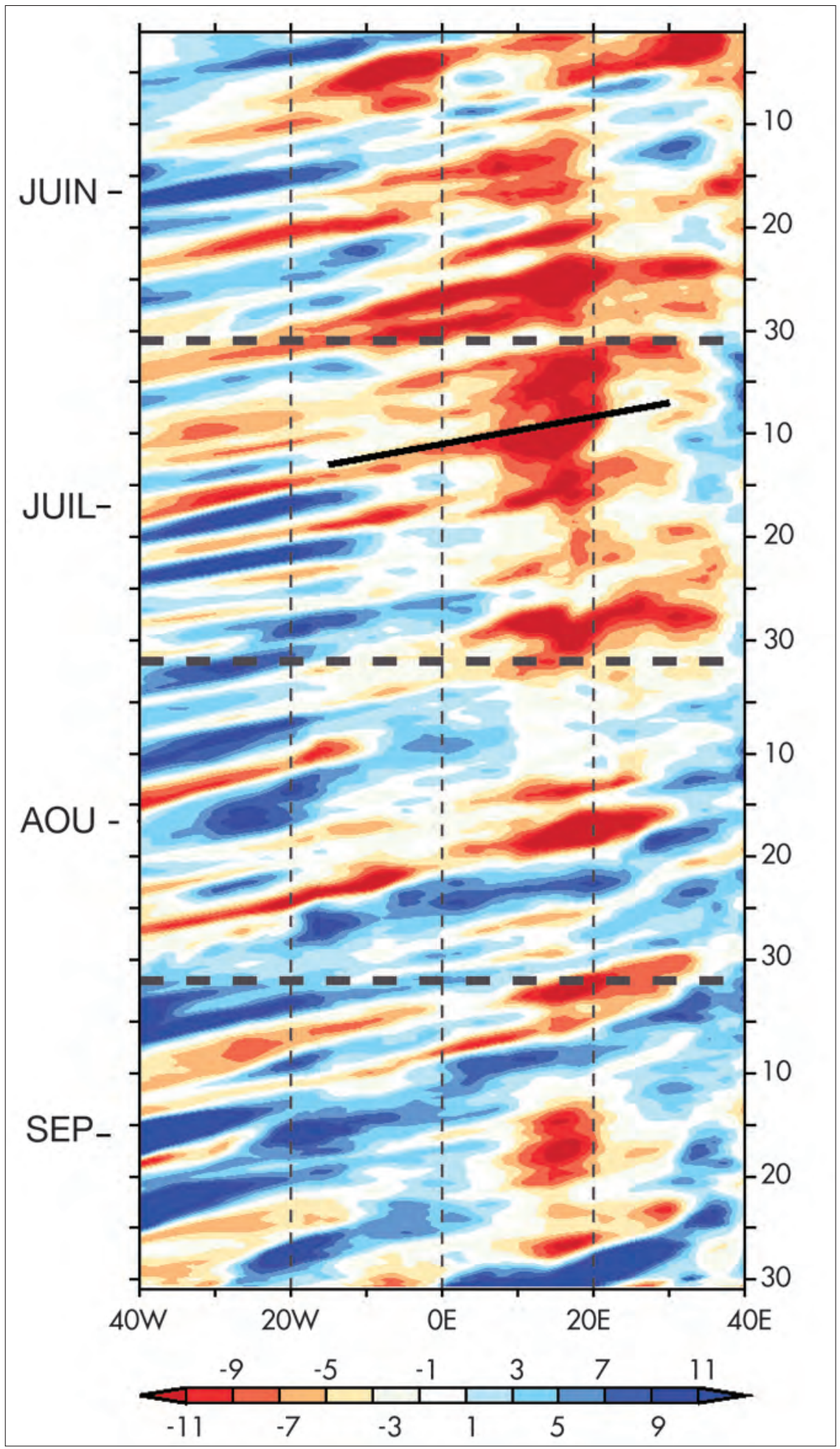

retardé le déclenchement de la mousson et, d'autre part, le passage d'une autre onde de Kelvin fin août 2006 entrant en interaction avec une onde d'est initiatrice du cyclone Debby sur l'Atlantique (Ventrice et al., 2012). Ces ondes, qui ont aussi été détectées le reste de l'année sur la côte guinéenne et l'Afrique Centrale (Nguyen et Duvel, 2008), apparaissent comme un système synoptique important pour la météorologie africaine dont l'analyse plus approfondie devrait apporter une opportunité d'améliorer la prévision opérationnelle.

\section{Émergence d'un modèle conceptuel dynamique de la MAO}

Le schéma conceptuel de la figure 1 reste statique alors que les précipitations de la MAO, outre leur cycle saisonnier, sont extrêmement variables dans le temps et dans l'espace, quelles que soient les échelles considérées, locales ou régionales, diurnes, intrasaisonnières ou interannuelles. L'impact sur les populations et les économies est souvent dramatique, en particulier à l'échelle intrasaisonnière. L'étude de la variabilité intrasaisonnière de la MAO date des dix dernières années, avec l'identification des principaux modes de variabilité, associés à 3 gammes d'échelles : $\sim 40$ jours en lien avec l'oscillation de Madden-Julian dans la bande équatoriale (Janicot et al., 2009), 15 jours avec les ondes de Kelvin et les modes guinéen et sahélien (Mounier et al., 2008 ; Janicot et al., 2010), et 3-10 jours avec les ondes d'est déjà discutées. Fontaine et al. (ce numéro, p. 41-48) abordent ce type de variabilité. Nous insistons ici sur l'émergence d'un modèle conceptuel plus dynamique de la MAO, ouvrant la voie à la compréhension et la prévision de sa variabilité intrasaisonnière.

À des échelles d'une quinzaine de jours, les travaux de Chauvin et al. (2010) et Lavaysse et al. (2010) ont montré que la DTS apparaît comme une courroie de transmission entre les latitudes tempérées et 1'Afrique. Elle tend à osciller entre deux configurations extrêmes, illustrées sur la figure 5. Le 7 juin 2011 est caractérisé par un fort événement, appelé DTS Est, avec une DTS très étendue, intense et une position anormalement nord-est, correspondant à une anomalie chaude (rouge) sur la Méditerranée, couplée à 

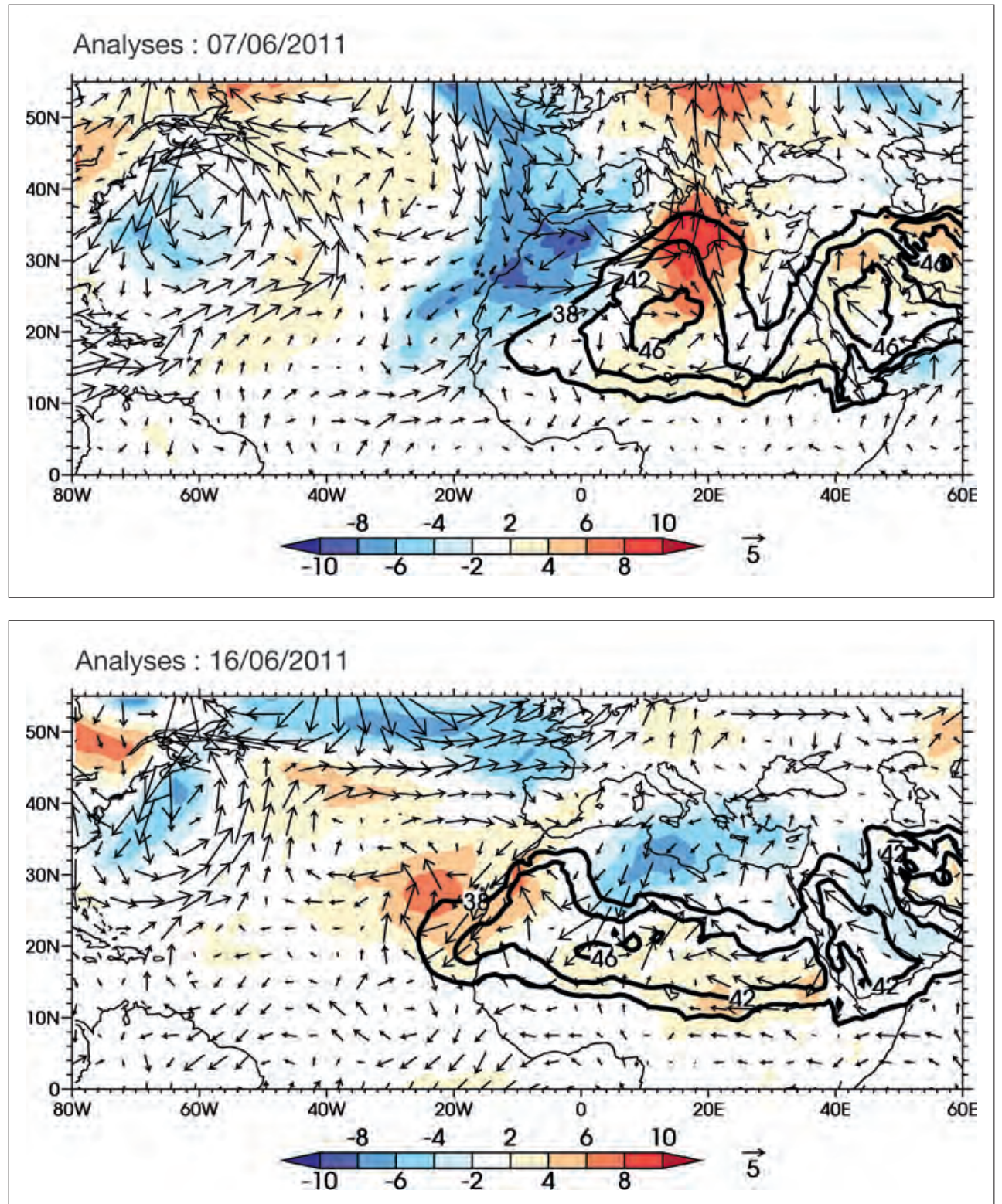

Figure 5 - Illustration du passage d'un régime DTS Est le 7 juin à un régime DTS Ouest le 16 juin 2011, pour les anomalies intrasaisonnières de température potentielle (couleur) et de vent (flèches) à $850 \mathrm{hPa}$. Les isolignes épaisses délimitent la zone de température de surface supérieure à $38{ }^{\circ} \mathrm{C}$ correspondant à la dépression thermique saharienne (DTS).

une anomalie de vent de sud en surface. La configuration inverse se produit sur le proche Atlantique avec une anomalie froide (bleue) venue du nord et repoussant vers 1'est la DTS. Cet événement DTS Est est intense avec des anomalies dépassant $10{ }^{\circ} \mathrm{C}$ et $10 \mathrm{~m} \mathrm{~s}^{-1}$. Dix jours plus tard, le 16 juin, la situation s'est inversée, avec un fort événement appelé DTS Ouest. Une ventilation froide de nord s'est installée sur la Méditerranée, repoussant la DTS vers l'ouest avec une anomalie chaude sur la côte marocaine. La climatologie montre que 3-4 événements de chaque type ont lieu pendant les 4 mois de la MAO. Ces événements sont précédés aux moyennes latitudes par une forte activité des ondes de Rossby sur l'Atlantique, détectées sur
(Janicot et al., 2010). Les mécanismes de propagation vers l'ouest et de modulation de la convection restent cependant encore à élucider.

\section{De nouveaux champs à explorer}

Cette brève synthèse met en valeur les progrès majeurs dans notre documentation et compréhension de la MAO et de ses processus clés, permis par le programme AMMA. Il a notamment eu l'originalité de mettre au premier plan les différents couplages entre les composantes du système : l'atmosphère, l'océan, les surfaces continentales, les aérosols, les nuages... AMMA ouvre ainsi de nouvelles pistes pour évaluer et améliorer la représentation de la MAO dans les modèles numériques de prévision du temps et du climat, encore peu performants sur l'Afrique (Karbou et al., dans ce numéro, p. 49-54; Hourdin et al., 2009).

Parmi les processus clés à étudier, la priorité va vers ceux impliqués dans le cycle de l'eau et de l'énergie. Nous avons vu que l'humidité conditionne fortement le comportement des surfaces continentales, de la couche limite, de la convection, des pluies et au final du bilan d'énergie sur l'Afrique. Il est donc essentiel de mieux observer, comprendre et modéliser le champ de vapeur d'eau qui indirectement va déterminer les ressources en eau que la mousson est à même de fournir à l'agriculture, l'élevage et plus largement aux activités humaines.

Méditerranée associée à un événement DTS Ouest atteint l'est du Sahel 4 jours plus tard, où elle réduit les précipitations. Cette anomalie négative des pluies se propage ensuite vers l'ouest traversant tout le Sahel en 4 jours, selon le scénario du mode sahélien, comme montré par Roehrig et al. (2011). Le scénario opposé se produit lors de l'occurrence d'un événement DTS Est avec une anomalie positive de précipitation traversant l'Afrique. Un autre lien a été établi avec l'onde de Rossby équatoriale
Partant d'un schéma statique quasi bidimensionnel, AMMA propose aujourd'hui une vision plus globale et dynamique de la MAO, faisant intervenir l'est de l'Afrique, les latitudes tempérées, les tropiques et la mousson indienne. Cette vision permet d'accéder à la variabilité du système, donnant espoir de pouvoir mieux le prévoir. Le chemin est encore long, mais les pistes ouvertes par AMMA et restant à explorer sont prometteuses. 